\title{
Extracolonic findings with the PillCam Colon: is panendoscopy with capsule endoscopy closer?
}

Authors

Institution
Javier Romero-Vázquez, Ángel Caunedo-Álvarez, Alba Belda-Cuesta, Victoria Alejandra Jiménez-García, Francisco Pellicer-Bautista, Juan Manuel Herrerías-Gutiérrez

Gastroenterology Service, Virgen Macarena University Hospital, Seville, Spain submitted $\quad$ 4. July 2015 accepted after revision 29. July 2016

\section{Bibliography} Dol http://dx.doi.org/ 10.1055/s-0042-115406 Published online: 14.9.2016 Endoscopy International Open 2016; 04: E1045-E1051

(c) Georg Thieme Verlag KG Stuttgart · New York E-ISSN 2196-9736

Corresponding author Javier Romero Vázquez, MD Gastroenterology Service Virgen Macarena University Hospital Avenida Dr. Fedriani s/n Seville 40171 Spain

Fax: +34-955-008805 javiromerov@gmail.com
Background and study aims: Colon capsules display images from the moment they are ingested, making the study of other extracolonic areas possible. The aim of this study was to analyze the significance of these extracolonic findings.

Patients and methods: In this single-center, prospective study, 165 patients underwent colon capsule endoscopy (CCE) between September 2009 and October 2012 to rule out colonic pathology. Images were recorded, without interruptions, from the moment the capsule was ingested until its battery ran out. The study was deemed complete when the capsule had traveled from the esophagus to excretion or until the hemorrhoidal plexus was observed.

Results: CCE was used for colorectal cancer (CRC) screening (81.2\%), to investigate for chronic diarrhea (9.7\%) and chronic iron deficiency anemia

\section{Introduction \\ $\nabla$}

Several studies have reported the usefulness of colon capsules (PillCam Colon; Given Imaging, Yoqneam, Israel, now Medtronic, Inc.), both the initial prototype (CCE-1) and later version (CCE-2), in the detection of colonic polyps in patients with a high risk of colorectal carcinoma (CRC) [1 -6]. In addition, the ability to observe the mucosa using the capsule may help in assessing the activity and extent of colonic inflammatory bowel disease (IBD). Colon capsule endoscopy (CCE) could also be complementary to colonoscopy in the case of incomplete colonoscopy, or could be used for patients with contraindications to sedation or anesthesia, or if a patient refuses to undergo a colonoscopy [7-11].

The life of the capsule's batteries and its ability to record images from the moment of its ingestion have allowed lesions to be observed at all levels of the digestive tract and, in some cases, relevant pathology to be identified, potentially meaning a
(6.1\%), and for patients with incomplete colonoscopy (3.0\%). The capsule returned findings in the esophagus in $52.1 \%$ of patients, in the stomach in $45.5 \%$ of patients, and in the small bowel in $70.7 \%$ of patients, with the findings being considered relevant in $4.9 \%, 9.7 \%$, and $22.6 \%$ of patients, respectively. The whole extent of the digestive tract was fully recorded in $86.1 \%$ of patients and the $\mathrm{Z}$ line could be fully observed in $57.6 \%$ of patients. There were no adverse events.

Conclusions: CCE allows the recording of images from almost the whole extent of the digestive tract in most patients, enabling relevant pathologies to be identified in extracolonic areas, particularly the small bowel. Technical and procedural improvements are still necessary in order to achieve better observation of the stomach and esophagus.

change in the way patients are clinically monitored.

To date, the probability of finding extracolonic pathologies at different levels of the digestive tract (esophagus, stomach, or small bowel), as well as the clinical relevance of any lesions found and their impact, in patients undergoing CCE to rule out colonic pathology, have not yet been evaluated. We therefore decided to perform a single-center, prospective, descriptive study with the primary aim of assessing the incidence and significance of extracolonic pathologies in patients undergoing CCE for various indications. Our secondary aims were to determine the percentage of patients in whom the whole extent of the digestive tract (from the esophagus to the hemorrhoidal plexus) could be fully recorded and the percentage of patients in whom the $\mathrm{Z}$ line could be fully observed. 


\section{Materials and methods}

$\nabla$

This prospective study of 165 patients undergoing CCE for screening/investigation of possible colonic pathology was performed between September 2009 and October 2012.

The indications to undergo CCE examination were CRC screening $(n=134 ; 81.2 \%)$, chronic diarrhea $(n=16 ; 9.7 \%)$, chronic iron deficiency anemia $(\mathrm{Hb}<10 \mathrm{~g} / \mathrm{dL} ; \mathrm{n}=10 ; 6.1 \%)$ and incomplete colonoscopy due to poor pain tolerance in patients who refused deep sedation for a second colonoscopy $(n=5 ; 3.0 \%)(\checkmark$ Table 1$)$. The following exclusion criteria were applied: age < 18; pregnancy; clinical, laboratory, or radiological suspicion of CRC (these patients underwent colonoscopy); clinical, laboratory, or radiological suspicion of an extracolonic pathology that would explain a patient's symptoms; suspected obscure gastrointestinal bleeding after positive fecal occult blood test; clinically overt gastrointestinal bleeding (presence of melena, hematochezia or rectal bleeding); as well as any of the established contraindications for capsule endoscopy examination.

Patients underwent standard preparation for the procedure as previously described (2L polyethyleneglycol administered the afternoon before the test, another $2 \mathrm{~L}$ on the day of the examination, with sodium phosphate [NaP] boosters used following preestablished indications) [4,5]. All patients swallowed the capsule in either the standing or sitting position, and remained in the Endoscopy Unit for a few minutes to check that there were no adverse effects after its administration.

Depending on the type of colon capsule used, one of the following procedures was chosen to enable the collection of images without interruption from the moment the capsule was ingested until its battery ran out: (a) CCE-C1: The capsule was extracted from the packaging and put into an envelope for 1 hour 50 minutes, obviating the initial period of inactivity that usually occurs to help save battery life. It was subsequently administered to the patient as described above. Once the capsule was found to have left the stomach, the first NaP booster $(30 \mathrm{~mL})$ was administered with $1 \mathrm{~L}$ water. If necessary, the second booster $(25 \mathrm{~mL})$ was administered 2 hours later. The recording continued without interruption from that moment until the capsule battery ran out. (b) CCE-C2: The data recorder was modified externally by pressing the center, left, and right buttons, one after the other, and then the right button again until the recorder was activated, thereby avoiding the usual hibernation period, and making the capsule believe, with its bidirectional interaction, that it was in the small bowel. Once the capsule was found to have left the stomach, boosters were administered following the same protocol as in CCE-1.

Based on their clinical significance, certain findings were considered to be 'clinically relevant' in their respective locations. (a) Esophagus: suspected intestinal metaplasia (Barrett's esophagus), esophageal varices, strictures, and neoplasms. (b) Stomach: active bleeding, adenomatous appearing polyps (not hyperplastic or inflammatory), peptic ulcer disease, angiodysplasias $>5 \mathrm{~mm}$, portal hypertensive gastropathy, and neoplasms. (c) Small bowel: active bleeding, angiodysplasias $>5 \mathrm{~mm}$, ulcerative enteropathy, suspicion of villous atrophy, erosive enteropathy, portal hypertensive enteropathy, polyps, and neoplasms. (d) Colon: polyps $>6 \mathrm{~mm}$, colorectal neoplasms, suspicion of IBD, diverticula with signs of diverticulitis, and angiodysplasias $>5 \mathrm{~mm}$. The following findings were considered 'non-clinically relevant' in their respective locations. (a) Esophagus: hiatal hernia, reflux esophagitis, Schatzki ring. (b) Stomach: non-adenomatous polyps, angiodys-
Table 1 Indications to undergo the PillCam Colon procedure.

\begin{tabular}{|lc|}
\hline Indication & Number (\%) \\
\hline CRC screening & $134(81.21)$ \\
\hline Chronic diarrhea & $16(9.70)$ \\
\hline Chronic iron deficiency anemia & $10(6.06)$ \\
\hline Incomplete colonoscopy & $5(3.03)$ \\
\hline
\end{tabular}

CRC, colorectal cancer.

plasias $<5 \mathrm{~mm}$, chronic superficial gastritis. (c) Small bowel: lymphangiectasia, angiodysplasias $<5 \mathrm{~mm}$, lymphoid nodular hyperplasia. (d) Colon: polyps $<6 \mathrm{~mm}$, diverticula without signs of diverticulitis, and angiodysplasias $<5 \mathrm{~mm}$.

Those patients with relevant findings after CCE (e.g. suspected intestinal metaplasia, adenomatous polyps, villous atrophy, etc.) underwent endoscopic procedures with the results being confirmed by biopsy.

Recordings or studies were deemed complete (panendoscopy) when the capsule traveled from the esophagus to excretion or, in the absence of excretion, until the hemorrhoidal plexus could be observed. Examination of the $\mathrm{Z}$ line was deemed complete when it could be fully observed. It was considered incomplete in all other cases or when remains or bubbles limited its assessment. The gastric, small-bowel, and colon transit times were also recorded (only in cases of complete studies).

Finally, we recorded the number of patients with colonic polyps and those showing relevant polyps (polyps $>6 \mathrm{~mm}$ ), and the degree of colonic cathartic preparation, which was divided into suitable (excellent, good) or inappropriate (fair, poor).

The study protocol was approved by the local ethics committee, and written informed consent was obtained from all patients who met the inclusion criteria and agreed to participate in the study.

\section{Results}

$\nabla$

This study included 165 patients ( 83 men, 82 women; mean age $56.39 \pm 16.76$ years). Findings were made by CCE in the esophagus for 86 patients (52.1\%), with the findings being considered relevant in eight patients (4.9\%): suspected intestinal metaplasia/ Barrett's esophagus $(n=5)$, esophageal varices $(n=2)$, and stenosis caused by a Schatzki ring $(n=1)(-$ Fig. 1$)$. Findings of hiatal hernia, esophagitis, non-Zenker's diverticulum, or Schatzki ring were considered to be irrelevant.

Findings were made by CCE in the stomach for 75 patients ( $45.5 \%$ ), with 16 patients $(9.7 \%$ ) having findings that were considered to be relevant: adenomatous-appearing polyps $(n=6)$, ulcers $(n=6)$, angiodysplasias $>5 \mathrm{~mm}(\mathrm{n}=2)$, portal hypertensive gastropathy $(n=2)$ Fig.2). Findings of non-erosive or non-ulcerative gastritis, hyperplastic appearing polyps, or angiodysplasia $<5 \mathrm{~mm}$ were considered to be irrelevant.

Findings were made by CCE in the small bowel of 116 patients (70.3\%), with 37 patients (22.4\%) having findings that were considered to be relevant: ulcers $(n=10)$, angiodysplasia $>5 \mathrm{~mm}(\mathrm{n}=$ $8)$, erosive enteropathy $(n=7)$, suspected villous atrophy $(n=6)$, polyps $(n=3)$, active bleeding $(n=2)$, and suspected Meckel's diverticulum $(\mathrm{n}=1)$ ( $\bullet$ Fig. 3 ). We categorized as non-relevant: isolated erosions, angiodysplasias $<5 \mathrm{~mm}$, mild duodenitis, lymphangiectasia, chyloma, xanthomas, or isolated diverticula. 

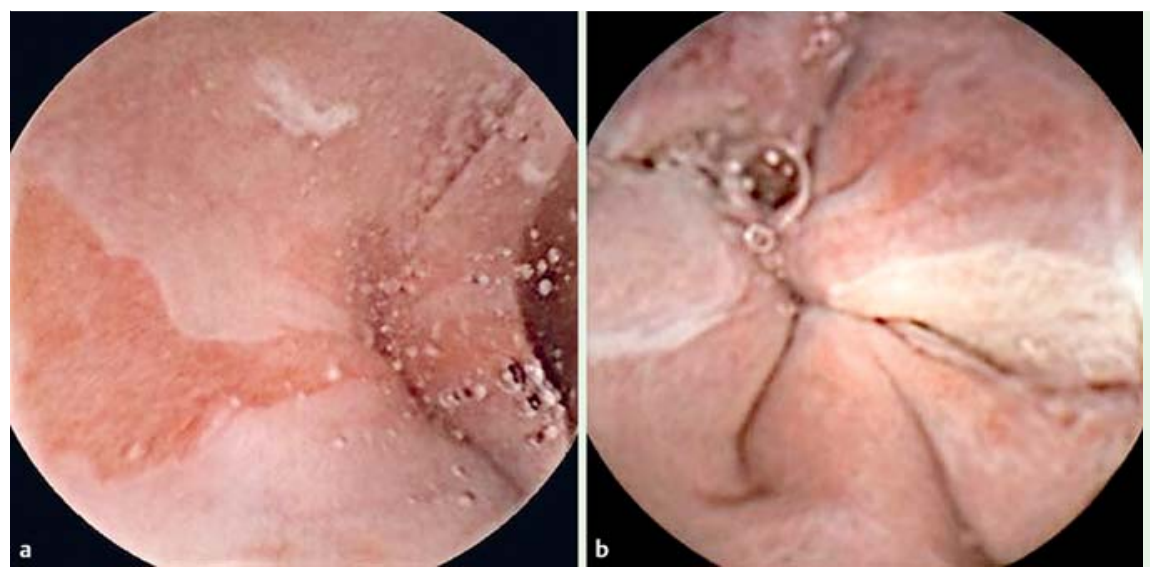

Fig. 1 a Capsule endoscopy image from the esophagus showing suspicion of Barrett's esophagus. b Capsule endoscopy image from the esophagus showing esophageal varices.
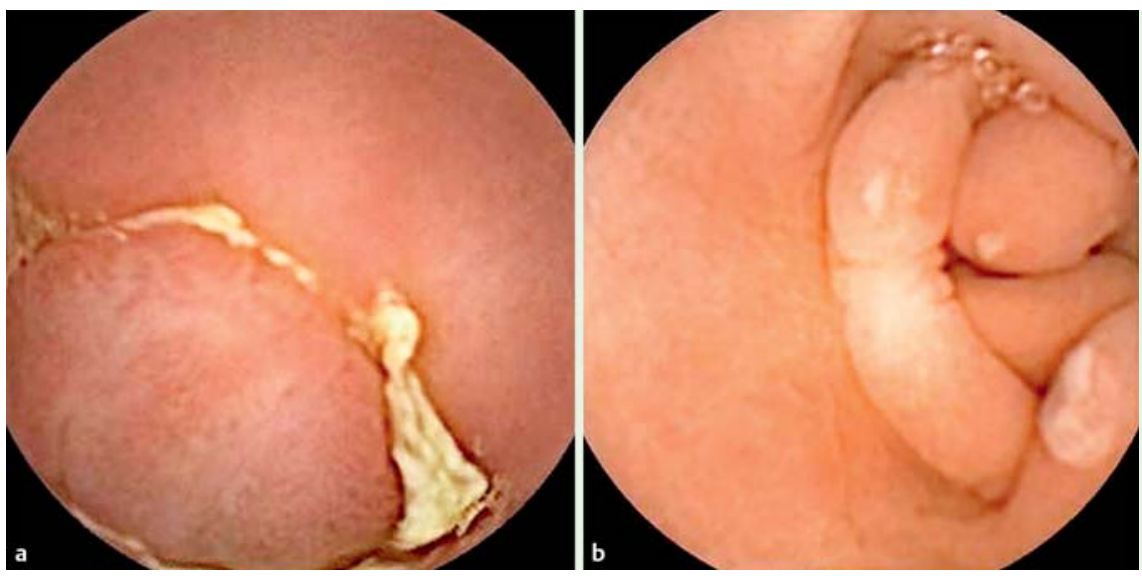

Fig. 2 a Capsule endoscopy image from the stomach showing adenomatous-appearing gastric polyps. b Capsule endoscopy image from the stomach showing gastric ulcers.

- Table 2 lists the findings made by the capsule in each of the extracolonic locations.

All patients with suspected significant extracolonic findings after CCE who required biopsies for histological confirmation (suspected intestinal metaplasia, adenomatous polyps, villous atrophy, etc.) underwent endoscopic studies that confirmed the CCE findings. CCE showed suspected intestinal metaplasia in five patients. All of these patients underwent gastroduodenoscopy with biopsy, which showed intestinal metaplasia without dysplasia, thereby establishing the diagnosis of Barrett's esophagus. The same occurred in patients with adenomatous appearing gastric polyps and in patients with suspected intestinal villous atrophy. The relationship between the indications for CCE and the relevant findings in each of the extracolonic locations is shown in - Table 3.

In total, 142 patients ( $86.1 \%$ ) had the whole extent of their digestive tract recorded. The capsule was excreted during the lifetime of its battery in $81.8 \%$ of patients. In $4.2 \%$ of patients, it was excreted with the battery depleted but after the hemorrhoidal plexus had been observed. In 23 patients (13.9\%), the study was considered incomplete as the capsule did not reach the hemorrhoidal plexus. At the time of battery depletion, the capsule was found to be in the stomach $(n=1)$, the small bowel $(n=2)$, the cecum $(n=1)$, the descending colon $(n=2)$, or in the majority of patients, between the sigmoid colon and rectum $(n=17)$ (৫ Table 4).

Incomplete study rates were not significantly different depending on whether the capsule administered was the CCE-C1 (15/ $113 ; 13.3 \%)$ or the CCE-C2 $(8 / 52 ; 15.4 \%)$. The $\mathrm{Z}$ line was fully observed $(100 \%$ of it) in $95 / 165$ patients $(57.6 \%)$. The mean transit time (range) for the stomach was 84.5 minutes (3- 728 minutes), for the small bowel it was 150.0 minutes (32- 308 minutes), and for the colon it was 148.1 minutes (6-860 minutes).

Colonic polyps were identified in $60 / 161$ patients $(37.3 \%)$, being significant $(>6 \mathrm{~mm}$ ) in 21 patients $(13.0 \%)$, with one patient being found to have a colorectal neoplasia ( $\bullet$ Fig.4). Among these 21 patients, the polyp sizes were $<10 \mathrm{~mm}(\mathrm{n}=10), 10-15 \mathrm{~mm}(\mathrm{n}=6)$, $16-20 \mathrm{~mm}(\mathrm{n}=4)$, and $>30 \mathrm{~mm}(\mathrm{n}=1$; located in the descending colon with the histology being adenocarcinoma). All of these patients underwent colonoscopy with polypectomy and no significant variations $( \pm 2 \mathrm{~mm})$ in polyp size were observed from the CCE findings. With the exception of the colorectal neoplasia, all of the polyps had a histology of tubular or tubulovillous adenoma with low grade dysplasia. There were no signs of angiodysplasia $>5 \mathrm{~mm}$, suspicion of colonic IBD, or other findings a priori considered to be significant.

The degree of colonic cleansing was adequate in 126 patients (78.3\%) and inadequate in 35 patients $(21.7 \%)$. There were no complications during or after the procedures.

\section{Discussion}

$\nabla$

Several published studies on the use of the PillCam Colon have demonstrated its usefulness in the detection of colonic polyps [1-6]. In addition, the number of studies addressing the usefulness of the CCE in other colonic diseases is increasing [7-11]. When analyzing the different colonic studies, we realized that, thanks to the ability of CCE to produce images of other extracolonic locations, it was able to identify lesions and pathologies 

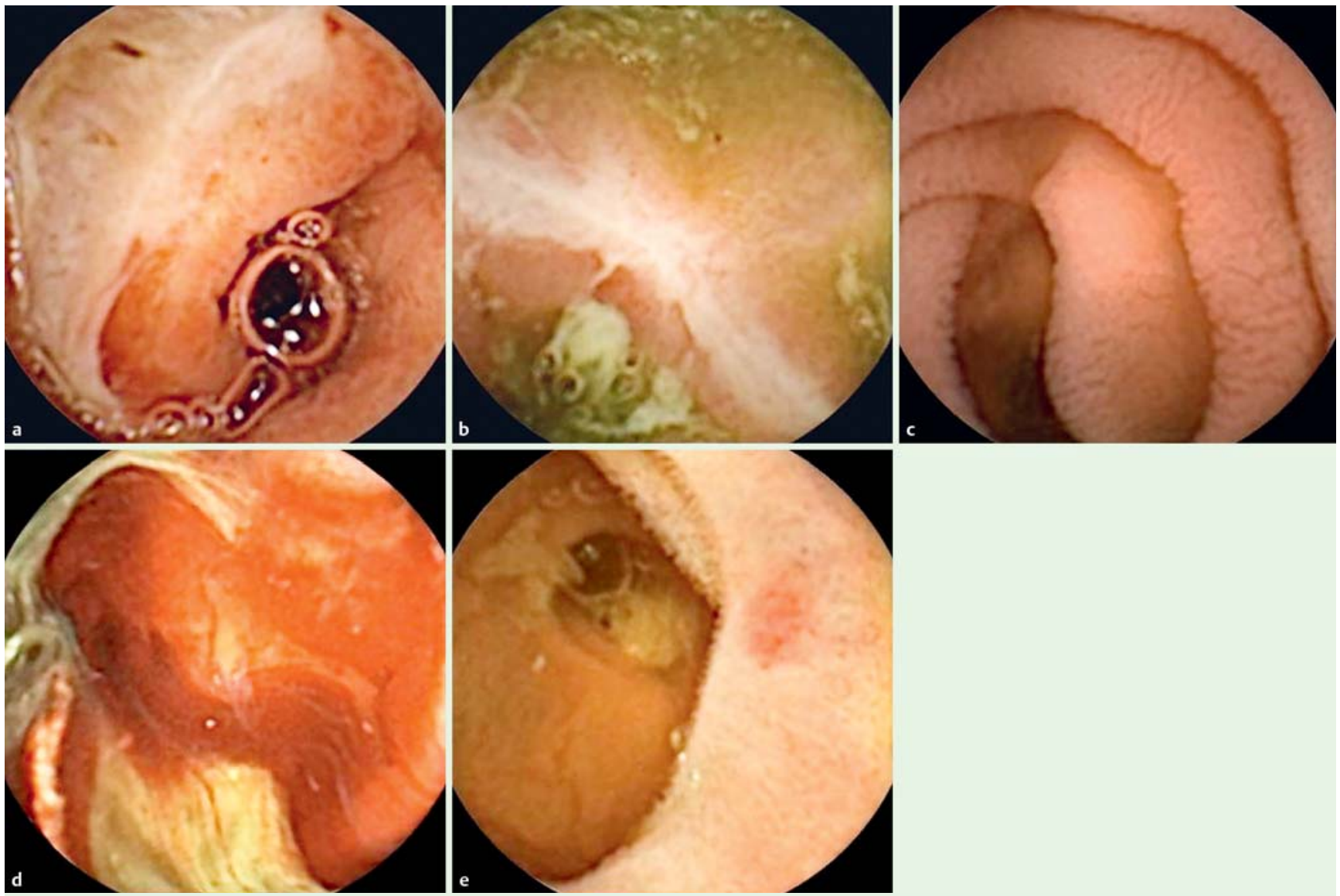

Fig. 3 a Capsule endoscopy image from the small bowel showing a large duodenal ulcer in a patient with anemia. b Capsule endoscopy image from the smal bowel showing an ileal ulcer in a patient later diagnosed with Crohn's disease. c Capsule endoscopy image from the small bowel showing suspected villous atrophy in a patient with diarrhea. $\mathbf{d}$ Capsule endoscopy image from the small bowel showing active bleeding of the jejunum and ileum in a patient with anemia. e Capsule endoscopy image from the small bowel showing a jejunal angiodysplasia in an asymptomatic patient.

\begin{tabular}{|c|c|c|c|}
\hline Location & Total findings, $\mathbf{n}(\%)$ & Relevant findings, n (\%) & Type of relevant findings \\
\hline Esophagus & $86(52.11)$ & $8(4.85)$ & $\begin{array}{l}\text { Barrett's esophagus }(n=5) \\
\text { Varices }(n=2) \\
\text { Stenosis }(n=1)\end{array}$ \\
\hline Stomach & $75(45.45)$ & $16(9.70)$ & $\begin{array}{l}\text { Ulcers }(n=6) \\
\text { Adenomatous polyps }(n=6) \\
\text { Angiodysplasias }>5 \mathrm{~mm}(n=2) \\
\text { Portal hypertensive gastropathy }(n=2)\end{array}$ \\
\hline Small bowel & $116(70.73)$ & $37(22.56)$ & $\begin{array}{l}\text { Ulcers }(n=10) \\
\text { Angiodysplasias }>5 \mathrm{~mm}(n=8) \\
\text { Erosive enteropathy }(n=7) \\
\text { Villous atrophy }(n=6) \\
\text { Polyps }(n=3) \\
\text { Active bleeding }(n=2) \\
\text { Suspected Meckel's diverticulum }(n=1)\end{array}$ \\
\hline
\end{tabular}

Table 2 PillCam Colon findings in each of the extracolonic locations.

within these areas [12]. There were, however, no studies available that stated the frequency of the findings observed in the different extracolonic areas and whether or not these were clinically relevant. Therefore, we decided to perform this descriptive study, with the main aim of analyzing the number of findings and their relevance in the esophagus, stomach, and small bowel.

After performing this analysis, we found that $22.6 \%$ of patients had clinically relevant small-bowel findings, an unexpectedly high percentage given that most patients were undergoing CCE for CRC screening and had previously been asymptomatic.
It could be argued that the presence of angiodysplasia $>5 \mathrm{~mm}$ should not be considered clinically relevant. Of the eight patients with angiodysplasia, two had anemia and no other cause for this was found, so we feel it should be considered relevant. The remaining six patients were asymptomatic, so one could be more critical in relation to its relevance. It is noteworthy that, in these patients, the angiodysplasias were not unique, ranging in number from three to nine, so they cannot be considered to be isolated lesions. If we took into consideration the classic study by Saurin et al. [13] in which lesions were divided according to their bleeding potential, these angiodysplasias $>5 \mathrm{~mm}$ could be classified as $\mathrm{P} 2$, 
Table 3 Relationship between the indications for patients to undergo the PillCam Colon procedure and relevant findings observed in each of the extracolonic locations.

\begin{tabular}{|c|c|c|c|c|}
\hline Indication & Number & Esophagus & Stomach & Small bowel \\
\hline CRC screening & 134 & 7 & 14 & 25 \\
\hline Chronic diarrhea & 16 & 0 & 0 & 5 \\
\hline $\begin{array}{l}\text { Chronic iron } \\
\text { deficiency anemia }\end{array}$ & 10 & 1 & 1 & 6 \\
\hline $\begin{array}{l}\text { Incomplete } \\
\text { colonoscopy }\end{array}$ & 5 & 0 & 1 & 1 \\
\hline Total & 165 & 8 & 16 & 37 \\
\hline
\end{tabular}

CRC, colorectal cancer.

Table 4 Percentage of complete and incomplete "panendoscopies". PillCam Colon location listed in incomplete cases.

\begin{tabular}{|c|c|c|}
\hline Panendoscopy ${ }^{1}$ & Number (\%) & Remarks \\
\hline Complete & $142(86.06)$ & $\begin{array}{l}\text { Excreted: } 81.82 \% \\
\text { Non-excreted: } 4.24 \%\end{array}$ \\
\hline Incomplete & $23(13.94)$ & $\begin{array}{l}\text { Location of the capsule when } \\
\text { the battery ran out: } \\
\text { Stomach }(n=1) \\
\text { Small bowel }(n=2) \\
\text { Cecum }(n=1) \\
\text { Descending colon }(n=2) \\
\text { Rectum-sigmoid colon }(n=17)\end{array}$ \\
\hline
\end{tabular}

1 Panendoscopy: Images recorded from the esophagus to the hemorrhoidal plexus or until the capsule was excreted.

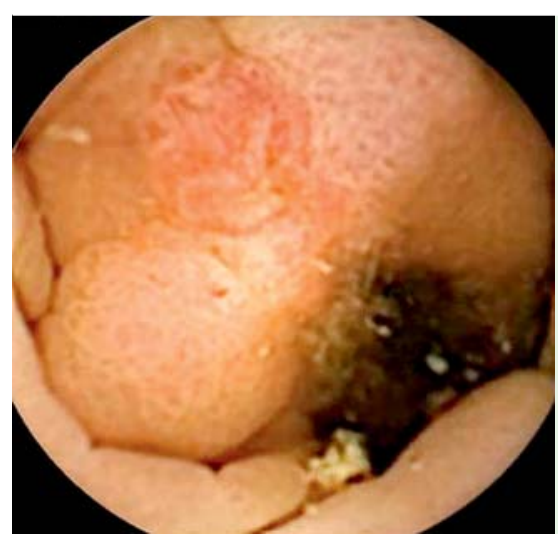

Fig. 4 Capsule endoscopy image from the colon showing a colorectal cancer.

i.e. high bleeding potential. In any case, and even if we excluded these six patients, a total of 31 patients had lesions in the small bowel, which means $18.9 \%$ of patients, almost one in five, undergoing CCE to screen for colonic pathology had relevant lesions identified in the small bowel.

In the subgroup of patients for whom the indication for CCE was CRC screening, we observed clinically relevant findings in extracolonic areas in 47 patients (34.3\%), 25 of these pathologies being located in the small bowel (18.7\%). Therefore, in our study, almost one in five patients undergoing CCE for CRC screening who did not have anemia or diarrhea (i.e. were asymptomatic) showed significant findings in the small bowel.

Among the 10 patients who underwent CCE for anemia, eight showed clinically relevant findings in extracolonic locations: esophageal varices and portal hypertensive gastropathy $(n=2)$, gastric polyp $(n=1)$, duodenal bulb ulcers $(n=1)$, suspected Meckel's diverticulum (not subsequently confirmed; $n=1$ ), small-bowel angiodysplasia $(n=1)$, active bleeding $(n=2)$. Of note, neither of the patients with active bleeding had previously shown obscure gastrointestinal bleeding, the results of the capsule endoscopy having been normal in one of them.

Among the 16 patients (31.3\%) who underwent CCE for diarrhea, five showed findings in the small bowel: capsule endoscopic pattern of villous atrophy $(n=3)$, small-bowel ulcers $(n=2)$. Three of the 10 patients with small-bowel ulcers had a history of nonsteroidal anti-inflammatory drug (NSAID) use and three were subsequently diagnosed with ileal or jejunal Crohn's disease (previous acute phase reactants and imaging tests were normal). No pathology was observed in the remaining four patients. As shown by previous studies, approximately $10-15 \%$ of healthy volunteers undergoing CCE show erosions or ulcers in the small bowel, the meaning of these lesions in asymptomatic subjects being uncertain, which could explain the presence of these ulcers in these last four patients $[14,15]$.

Patients with relevant findings in the esophagus and stomach (8 and 16 , respectively) underwent upper gastrointestinal endoscopies that confirmed the existence of Barrett's esophagus (after biopsies and histology had been performed) in all suspected cases, showed the size of varices (the same as was observed with $\mathrm{CCE}$ ), allowed the resection of gastric and adenomatous polyps (confirmed after histology), and allowed treatment of angiodysplasias with argon-beam coagulation. Balloon-assisted enteroscopies were performed in patients with significant findings in the small bowel $(n=37)$ : in those with ulcers suspicious of Crohn's disease without a history of NSAID intake $(n=3)$, and in those with significant angiodysplasias $(n=8)$ or active bleeding $(n=2)$ (the former treated with conservative or medical treatments and the latter with argon-beam coagulation).

After the CCE procedure, all patients with relevant findings in the esophagus, stomach, and small bowel had their previous diagnosis modified, irrespective of whether it was made after medical therapy (ulcerative gastroenteropathy, Crohn's disease, villous atrophy), endoscopic treatment (gastric polyps, angiodysplasias, active bleeding), or by changes in the clinical follow-up of patients (Barrett's esophagus). The extracolonic findings identified by the CCE changed the initial diagnosis in approximately onethird of all patients included in the study.

The attempt to perform a panendoscopy means that the CCE ingestion protocol had to be modified. With the CCE-C1, we would lose almost 2 hours of battery lifetime (as the capsule is administered to the patient 1 hour and 50 minutes after its activation) and with the $\mathrm{CCE}-\mathrm{C} 2$, we would avoid the hibernation period and we would thus save the battery.

Therefore, one might think that modifying the said protocol and consuming more battery power before the capsule reached the colon would increase the number of incomplete colon studies or the absence of capsules excreted. This was however not the case in our study, as the results of complete studies and capsules excreted during the life of the battery $(86.0 \%$ and $81.8 \%$, respectively) are very similar to those of other major studies published with CCE $[3,5]$. That is, in our series, the attempt to perform a panendoscopy did not result in a lower rate of complete colonic studies. Nevertheless, a $15 \%$ rate of incomplete studies is not a desirable rate and, in future, the rate of complete studies should be higher than $90 \%$ of procedures. For this reason, different preparative regimens are being studied by various groups with the aim of improving both colon propulsion and cleansing, as well as comfort for the patient, although the best results have not yet been achieved [16-18]. 
The low rate of detection of colonic polyps in our study (37.3\%), especially of those considered to be significant (>6 mm; $13.0 \%$ ), could be due to the fact that, unlike the major studies published on this subject, our patients were a low risk population, because patients with higher probabilities of having polyps or CRC were excluded from our study and underwent colonoscopy. Data relating to the percentage of patients with inadequate colonic preparation (22\%) in our study is similar to that found in other major studies published on CCE-C2 preparation, which have shown rates of suboptimal preparation of $19-22 \%[4,5]$, and higher in comparison to data from the main article on CCE-C1 [3]. However, we believe that these rates need to be improved in order to consider CCE for the study of colonic pathology. In this regard, studies with other preparative regimens, such as split dosing, should be considered in the future.

The PillCam Colon's ability to explore the entire small bowel may suggest the possibility of using it to attempt panendoscopy in different scenarios. The most clinically relevant scenarios might be: (1) assessment of IBD activity and extent, including its undetermined or unclassified forms, and atypical ulcerative colitis, as suggested in previous studies with PillCam SB [19,20]; (2) obscure gastrointestinal bleeding with previous incomplete colonoscopy; and (3) patients with anemia undergoing CCE to screen for colonic pathology. These potential indications should be considered purely speculative, given the lack of previous studies on the matter, and should be confirmed in prospective studies with a large number of patients.

In relation to the esophageal findings, the first thing to take into account is that the percentage of patients in whom the $\mathrm{Z}$ line could be fully observed was low $(<60 \%)$, so we can assert that CCE cannot assess the esophageal mucosa adequately, and therefore it was not possible to rule out underlying associated pathologies. In our opinion, an explanation for this low percentage could be that the ingestion protocol (with the patient in a standing or sitting position) is not appropriate for assessment of the esophagus because, as shown in previous studies, the percentage of patients in whom the $\mathrm{Z}$ line can be fully observed is higher when the capsule is ingested with the patient in the right lateral decubitus position [21-23]. Subsequent studies should verify whether ingestion in this position increases the percentage of patients in whom the $\mathrm{Z}$ line can be fully observed and whether the number of esophageal findings is higher; we believe that, in the future, this should be taken into account in patients undergoing CCE for CRC screening, given the possibility of a higher incidence of Barrett's esophagus in these patients, according to some authors [24-27].

In relation to the gastric findings, it seems clear that the PillCam Colon, like other known capsules (PillCam SB and PillCam ESO), is not the best option for a proper assessment of the gastric mucosa and it cannot in any case ensure a complete scan of the cavity. Extracorporeal management of the capsule by remote control, tested in experimental studies, may increase the percentage of the mucosa observed, although this needs to be carefully evaluated in controlled studies with larger numbers of patients [28-33]. Until this can be verified, upper gastrointestinal endoscopy will remain the gold standard for the observation of the gastric mucosa and any potential pathologies.

This study has some limitations. First, the study population selected was heterogeneous because the indications we included were CRC screening, chronic diarrhea, iron deficiency anemia, and incomplete colonoscopy (the population selected needed to cover these indications). However, we believe that the use of the capsule on this wider group of patients is an advantage.

Another possible limitation is that we used two types of CCE (the $\mathrm{C} 1$ and $\mathrm{C} 2$ versions). This was because the second generation of the colon capsule emerged while we were developing this study and its new features allowed us to make new findings in a way more comfortable for patients than the first. The use of the new capsule did not have any effect on cathartic preparation or boosters because these were administered once the capsule had been found to have left the stomach. Therefore, although the system changed, neither the preparation nor the number of complete explorations were affected.

Finally, it should be noted that one of the main features of CCE is that it may avoid conducting more invasive tests such as colonoscopy. Therefore, it is a useful test when a patient refuses colonoscopy under anesthesia, in cases of incomplete colonoscopy, or when there are contraindications to sedation or anesthesia for the patient.

\section{Conclusions}

$\nabla$

In short, in view of the results obtained in our study and exercising caution, given the absence of previously published data, the PillCam Colon allows almost the entire digestive tract to be recorded in most patients, thereby enabling relevant pathologies to be found in other sections of the digestive tract, particularly the small bowel. Technical and procedural improvements are still necessary to achieve better observation of the stomach and esophagus.

\section{Competing interests: None}

\section{References}

1 Eliakim R, Fireman Z, Gralnek IM et al. Evaluation of the PillCam Colon capsule in the detection of colonic pathology: results of the first multicenter, prospective, comparative study. Endoscopy 2006; 38: 963 -970

2 Schoofs N, Devière J, Van Gossum A. PillCam colon capsule endoscopy compared with colonoscopy for colorectal tumor diagnosis: a prospective pilot study. Endoscopy 2006; 38: 971 - 977

3 Van Gossum A, Munoz-Navas M, Fernandez-Urien I et al. Capsule endoscopy versus colonoscopy for the detection of polyps and cancer. NEJM 2009; 361: $264-270$

4 Eliakim R, Yassin K, Niv $Y$ et al. Prospective multicenter performance evaluation of the second-generation colon capsule compared with colonoscopy. Endoscopy 2009; 41: 1026-1031

5 Spada C, Hassan C, Munoz-Navas M et al. Second-generation colon capsule endoscopy compared with colonoscopy. Gastrointest Endosc 2011; 74: 581-589

6 Spada C, Hassan C, Galmiche JP et al. Colon capsule endoscopy: European Society of Gastrointestinal Endoscopy (ESGE) guideline. Endoscopy 2012; 44: 527-536

7 Sung J, Ho KY, Chiu HM et al. The use of Pillcam Colon in assessing mucosal inflammation in ulcerative colitis: a multicenter study. Endoscopy 2012; 44: $754-758$

8 Ye CA, Gao YJ, Ge ZZ et al. PillCam COLON capsule endoscopy versus conventional colonoscopy for the detection of the severity and extent of ulcerative colitis. J Dig Dis 2012; 14: 117-124

9 Meister T, Heinzow HS, Domagk D et al. Colon capsule endoscopy versus standard colonoscopy in assessing disease activity of ulcerative colitis: a prospective trial. Tech Coloproctol 2013; 17: 641-646

10 Pioche $M$, de Leusse A, Filoche B et al. Prospective multicenter evaluation of colon capsule examination indicated by colonoscopy failure or anesthesia contraindication. Endoscopy 2012; 44: 911-916

11 Alarcón-Fernández 0, Ramos-López L, de Ganzo ZA et al. Effects of colon capsule endoscopy on medical decision-making in patients with incomplete colonoscopies. Clin Gastroenterol Hepatol 2012; 11: $534-$ 540 
12 Karagiannis S, Dücker C, Dautel P et al. Identification of the duodenal papilla by colon capsule endoscope. Z Gastroenterol 2010; 48: 753 755

13 Saurin JC, Delvaux M, Gaudin JL et al. Diagnostic value of endoscopic capsule in patients with obscure digestive bleeding: blinded comparison with video push-enteroscopy. Endoscopy 2003; 35: 576-784

14 Goldstein JL, Eisen GM, Lewis B et al. Video capsule endoscopy to prospectively assess small bowel injury with celecoxib, naproxen plus omeprazole, and placebo. Clin Gastroenterol Hepatol 2005; 3: 133 141

15 Caunedo-Alvarez A, Gómez-Rodríguez BJ, Romero-Vázquez J et al. Macroscopic small bowel mucosal injury caused by chronic nonsteroidal anti-inflammatory drugs (NSAID) use as assessed by capsule endoscopy. Rev Esp Enferm Dig 2010; 102: 80-85

16 Ramos L, Alarcón O, Adrian $Z$ et al. One-day versus two-day cleansing for colon capsule endoscopy: a prospective randomized pilot study. Gastroenterol Hepatol 2014; 37: 101 - 106

17 Kakugawa $Y$, Saito $Y$, Saito $S$ et al. New reduced volume preparation regimen in colon capsule endoscopy. World J Gastroenterol 2012; 18: $2092-2098$

18 Hartmann $D$, Keuchel $M$, Philipper $M$ et al. A pilot study evaluating a new low-volume colon cleansing procedure for capsule colonoscopy. Endoscopy 2012; 44: $482-486$

19 Maunoury V, Savoye G, Bourreille A et al. Value of wireless capsule endoscopy in patients with indeterminate colitis (inflammatory bowel disease type unclassified). Inflamm Bowel Dis 2007; 13: 152 - 155

20 Mehdizadeh S, Chen G, Enayati PJ et al. Diagnostic yield of capsule endoscopy in ulcerative colitis and inflammatory bowel disease of unclassified type (IBDU). Endoscopy 2008; 40: $30-35$

21 Gralnek IM, Rabinovitz R, Afik D et al. A simplified ingestion procedure for esophageal capsule endoscopy: initial evaluation in healthy volunteers. Endoscopy 2006; 38: 913-918

22 De Jonge PJ, Van Eijck BC, Geldof $H$ et al. Capsule endoscopy for the detection of oesophageal mucosal disorders: a comparison of two different ingestion protocols. Scand J Gastroenterol 2008; 43: 870-877
23 Fernandez-Urien I, Borobio E, Elizalde I et al. Z-line examination by the PillCam SB: prospective comparison of three ingestion protocols. World J Gastroenterol 2010; 16: 63-68

24 Howden $C W$, Hornung CA. A systematic review of the association between Barrett's esophagus and colon neoplasms. Am J Gastroenterol 1995; 90: $1814-1819$

25 Ward EM, Wolfsen HC, Achem SR et al. Barrett's esophagus is common in older men and women undergoing screening colonoscopy regardless of reflux symptoms. Am J Gastroenterol 2006; 101: 12-17

26 De Jonge PJ, van Blankenstein M, Looman CW et al. Risk of colorectal cancer in patients with Barrett's esophagus: A Dutch population-based study. Am J Gastroenterol 2010; 105: 77-83

27 Bolino MC, Caro L, Cerisoli C. Barrett's esophagus: is it a risk factor for colorectal cancer or an association between them does exist? Am J Gastroenterol 2011; 106: 169

28 Swain $P$, Toor A, Volke $F$ et al. Remote magnetic manipulation of a wireless capsule endoscope in the esophagus and stomach of humans (with videos). Gastrointest Endosc 2010; 71: 1290-1293

29 Keller J, Fibbe C, Volke F et al. Remote magnetic control of a wireless capsule endoscope in the esophagus is safe and feasible: results of a randomized, clinical trial in healthy volunteers. Gastrointest Endosc 2010; 72: 941 - 946

30 Van Gossum A, Ibrahim M. Video capsule endoscopy: what is the future? Gastroenterol Clin North Am 2010; 39: 807-826

31 Keller J, Fibbe C, Volke $F$ et al. Inspection of the human stomach using remote-controlled capsule endoscopy: a feasibility study in healthy volunteers (with videos). Gastrointest Endosc 2011; 73: 22-28

32 Lien GS, Liu CW, Jiang JA et al. Magnetic control system targeted for capsule endoscopic operations in the stomach - design, fabrication, and in vitro and ex vivo evaluations. IEEE Trans Biomed Eng 2012; 59 : $2068-2079$

33 Keller J, Fibbe C, Rosien $U$ et al. Recent advances in capsule endoscopy: development of maneuverable capsules. Expert Rev Gastroenterol Hepatol 2012; 6: $561-566$ 